Ann. Abeille, I962, 5 (3), 20I-213

\title{
TECHNIQUES D'ÉTUDE DES POPULATIONS D'INSECTES POLLINISATEURS
}

\author{
Jacques LECOMTE \\ Station de Recherches sur l'Abeille it les Insectes socianx, \\ Bures-sur-Yvette (Seine-et-Oise.)
}

SOMMAIRE

L'étude des populations d'insectes pollinisateurs, essentiellement composées d'Apides, nécessite l'emploi d'un certain nombre de techniques. Celles qui sont étudiées dans ce travail se rapportent essentiellement à l'appréciation de la densité de la population et au marquage individuel ou collectif d'individus faisant partie de ces populations.

\section{INTRODUC'TION}

Au cours de ces dernières années, un nombre croissant de recherches ont été effectuées dans différents pays sur les problèmes posés par la pollinisation des plantes cultivées.

L'intérêt économique de ces questions paraît de premier plan, puisque différents auteurs chiffrent la plus value apportée à l'agriculture par l'activité pollinisatrice de l'Abeille domestique, à dix ou quinze fois la valeur du miel et de la cire. Aux essais empiriques effectués dans les premiers temps de l'étude de ces problèmes, succèdent de plus en plus des recherches de base. Pour assurer en toute circonstance une pollinisation satisfaisante de plantes aussi variées que celles qui sont contenues dans les listes de végétaux bénéficiant d'un apport de pollen par l'insecte, il est indispensable de connaître bien des points de la biologie des pollinisateurs. Il sera essentiellement indispensable de pouvoir répondre avec exactitude aux questions d'ordre éthologiques et écologiques qui se posent lors de l'utilisation pratique. Ces recherches de base nécessitent évidemment l'utilisation de techniques spéciales. Ces techniques sont celles des " naturalistes de terrain " et diffèrent beaucoup de celles qui sont utilisées au laboratoire. Elles ont, certes, souvent fait l'objet de publications, mais rarement d'une revue systématique. Le présent travail s'attaque 
à cette tâche, il sera sans doute suivi de présentations similaires de techniques. permettant d'effectuer l'étude éthologique ou écologique des insectes pollinisateurs. Parmi ces insectes, je me limiterai aux Apides; d'abord parce que je n'ai personnellement pas étudié d'autres groupes, ensuite parce que ces insectes représentent certainement au moins go p. roo de la faune pollinisatrice d'intérêt économique.

\section{LE DÉXOMBREMENT DES POPULATIONS}

Une première question se pose : comment pouvons-nous estimer soit l'effectif total de la population présente sur un champ, soit l'effectif total de la population d'une espèce dans une région délimitée?

1)ans le premier cas, étant donné la taille relativement importante de la plupart des Apides, il semble qu'il soit possible d'admettre, comme référence de base, 1e comptage à vue. Deux méthodes semblent possibles : la première est celle qui consiste à observer une superficie réduite, généralement de l'ordre du mètre carré, pendant un temps assez long, la seconde consiste en un dénombrement instantané effectué sur une surface beaucoup plus importante.

Pour la majorité des auteurs, la deuxième méthode semble plus intéressante en effet, très généralement, les hétérogénéités dans l'espace sont plus importantes que les hétérogénéités dans le temps, même quand les surfaces sont petites, de 1'ordre de 1'hectare. En ce qui concerne les hétérogénéités dans le temps, un certain nombre de remarques doivent être faites:

Io Elles peuvent être le fait des facteurs météorologiques ;

$2^{\circ}$ Elles peuvent être dues à des facteurs de comportement propre à l'insecte considéré ;

$3^{0}$ Elles peuvent provenir du végétal ou des végétaux butinés.

Je donnerai quelques exemples : en ce qui concerne le premier groupe, il ne faut pas penser que de minimes variations dans les conditions météorologiques aient une influence très sensible sur le nombre d'individus présents sur le champ.

Seules les modifications relativement importantes : pluie, vent au-dessus de $20 \mathrm{~km} / \mathrm{h}$, température en dessous de $20^{\circ}$, sont à prendre en considération. De même, d'une heure après le lever du soleil à une heure avant, toutes choses étant égales par ailleurs, il est possible de considérer que les variations de luminosité ne jouent pas un rôle important.

Ces différentes considérations ne sont évidemment valables que pour les mois de printemps et d'été.

Les facteurs appartenant au deuxième groupe peuvent être très divers. Je me contenterai de signaler la "pause de midi " des Abeilles. Bien que l'on ait cherché à relier cet arrêt de travail du milieu de la journée à des facteurs météorologiques ou à une baisse de sécrétion nectarifère, de récents travaux montrent qu'elle est encore sans explications (HAMbI,ETON I95I, ScHUA r952).

Nous en arrivons au troisième groupe de facteurs, ceux qui sont liés au végétal. Chez certaines espèces et dans certaines conditions, la sécrétion nectarifère est régulière et suffisamment abondante toute la journée, pour d'autres, au contraire, il peut n'y avoir sécrétion que le matin. 
On comprend aisément que dans le premier cas, les comptages effectués le matin et l'après midi seront comparables, tandis que, dans le second cas, il n'en sera rien. Il faut évidemment tenir compte des très nombreux cas intermédiaires.

Je ne prendrai que quelques exemples observés personnellement. La sécrétion nectarifère $d u$ Tournesol est régulière, aussi, dans une série de comptages effectués le matin sur I5 900 capitules, trouve-t-on une moyenne de $\mathbf{r}, 08$ butineuses par capitule, tandis que l'après-midi, un comptage effectué sur le même nombre de capitules donne tune moyenne de I, I5 Abeilles par capitule.

Pour des raisons diverses, le Tilleul après huit heures du matin, le Sarrazin après I2 heures, les Cistes après $I_{3}$ heures peuvent être totalement désertés.

I a récolte du pollen a été spécialement bien étudiée à cet égard (PERcival, I950-55, MaUrizio I953). Pour ce dernier auteur, il existe au moins trois types de récoltes : récolte matinale, récolte pendant toute la journée, récolte 1'après-midi seulement.

En ce qui concerne les hétérogénéités de la distribution dans l'espace, toute une autre série de remarques peut être faite :

a) Nous pouvons nous demander si le champ étudié est homogène en ce qui concerne les facteurs attractifs. Par exemple, nombre de fleurs ouvertes, quantité et qualité du nectar; l'expérience montre qu'il ne l'est jamais.

b) Nous devons connaître les facteurs de comportement qui sont susceptibles de rentrer en jeu; pour l'Abeille, par exemple : effet répulsif de l'ombre, du relief, rôle des " chemins de butinage ", etc.

Parmi ces facteurs de comportement, on notera que si la densité de populations en ce qui concerne les Abeilles domestiques, est d'autant plus grande qu'on se rapproche du rucher, il y a cependant une accumulation de butineuses à l'extrémité distale du champ. Mais, ici encore, il ne s'agit pas d'une loi générale. De toute manière, il sera bon de se méfier des comptages effectués sur les bordures. Outre cet effet, il existe souvent une forte hétérogénéité entre les végétaux de bordure et ceux qui se trouvent au centre du champ. Parfois, cependant, cette différence peut exister en sens inverse, parfois elle est très peu sensible. Je donnerai comme exemple la différence entre les moyennes d'un comptage effectué sur 20 noo capitules de Tournesol situés en bordure et Io ooo capitules situés au centre. Pour les bordures, nous avons observé I,I 8 Abeilles par capitule, pour le centre $\mathrm{I}, 37$.

Après ces quelques considérations, nous pouvons nous attarder quelques instants à considérer quelles sont les méthodes généralement utilisées pour effectuer les comptages sur le terrain. Étant bien entendu que les procédés consistant à observer pendant longtemps une surface réduite sont à éliminer. Une méthode souvent employée est celle qui consiste à délimiter de petites surfaces réparties au hasard sur le champ et à effectuer des comptages extemporanés plusieurs fois par jour sur ces surfaces.

VANSELI, et TODD (I946) ont utilisé des surfaces d'un Yard carré pour étudier la pollinisation de la Iuzerne, JAmiesox (I950) des surfaces de deux Yards carrés dans un champ de Trèfle violet. SMith et TOwnsend (I952) ont fait de pertinentes critiques à cette méthode. Il semble, en effet difficile, surtout pour les plantes à floraison échelonnée dans le temps, de ne pas piétiner la végétation autour des points de comptage. On aboutit ainsi à la création d'îlots de plantes fleuries, très spécialement attractifs et les fréquences observées sont supérieures à celles qui existent sur l'ensemble du champ. 
Ces mêmes auteurs ont proposé l'utilisation d'un compteur permettant de délimiter facilement des aires d'une superficie choisie à l'avance. Il s'agit essentiellement d'un prisme monté à l'extrémité d'un support extensible. Un réticule divise la superficie choisie en quatre parties afin de faciliter le comptage qui se fait en regardant à travers le prisme dont le champ de vision est, dans le cas présent, d'un Yard carré. Un observateur peut ainsi effectuer 45 dénombrements par heure, en choisissant une durée d'observation de Io secondes. Ia seule limite d'utilisation se trouve être la hauteur des plantes observées; sans l'adjonction d'un piédestal pour l'observateur, il est difficile d'observer des plantes de plus de $75 \mathrm{~cm}$. De même, il est quasi impossible de déterminer les insectes ainsi comptés.

J'ai personnellement employé une technique différente. Je décris dans le champ, il s'agit de luzerne, un certain nombre de bandes d'une centaine de mètre de longueur. Une baguette d'un mètre de longueur est tenue à la main à la hauteur des fleurs et je compte au passage les insectes compris entre ma main et l'extrémité de la baguette. Le comptage est inscrit au fur et à mesure sur un compteur à main. La baguette peut être avantageusement terminée par un filet, ce qui permet de capturer les insectes dont on veut effectuer la détermination précise. Selon la densité de la couverture végétale, cette opération demande de 5 à 15 secondes par mètre carré. Parfois, le repérage se fait aussi bien à l'oreille qu'avec la vue, en particulier certaines espèces d'Apides sauvages affectionnent les fleurs situées en position basse et sont très difficiles à apercevoir.

Il est bien certain qu'une méthode universelle n'existe pas. Elle doit être adaptée au végétal considéré. En ce qui concerne le Tournesol, un comptage par capitule sur une centaine de capitules situés l'un après l'autre semble une méthode satisfaisante. Dans le cas des arbres fruitiers, l'unité de référence la plus généralement adoptée est l'arbre. Un certain nombre d'arbres sont choisis et l'observateur tourne autour de chacun en notant le nombre d'insectes visibles. Quand l'arbre a une taille importante, l'observation des branches supérieures doit se faire à l'aide d'une paire de jumelles. Cette méthode a été en particulier employée par VANSEI, (I942). I)'autres auteurs ont cherché à remplacer cette observation qui n'est pas sans défauts par divers procédés de récolte des échantillons.

Le filet fauchoir a souvent été proposé dans ce but (LINSLEY, I946). Indiscutablement cet instrument ramasse un grand nombre d'insectes en très peu de temps, mais il est permis de se demander quelle est la signification de cet échantillonnage.

Tout d'abord, il est assez difficile de standardiser le coup de filet. Il peut faucher une plus ou moins grande surface, à une altitude légèrement variable, à une vitesse qui n'est pas uniforme. D'un individu à un autre et aussi d'un coup de filet à l'autre, les différences peuvent être importantes.

Enfin, il semble que les différentes espèces d'insectes ne se laissent pas capturer au fauchoir avec la même bonne volonté. Je citerai un exemple personnel portant sur la faune pollinisatrice de la Luzerne. Tandis qu'une observation à vue m'avait donné une proportion de 8,75 Rophites canus pour zoo Apis mellifica, le filet fauchoir dans le même champ, ne me laissait capturer qu'un Rophites pour roo Abeilles. Une observation du comportement de ces deux insectes permet de comprendre l'infériorité du fauchoir. Tandis que l'Abeille butine de préférence les fleurs situées en haut des tiges et ne s'enfonce que peu dans la masse de la végétation, Rophites 
canus, Apide d'une taille égale au I/3 de l'Abeille, butine de préférence les fleurs situées en dessous du niveau supérieur de la végétation.

En l'occurence, il s'agissait d'une Luzerne ayant une hauteur moyenne de $80 \mathrm{~cm}$, il n'était donc pas possible d'atteindre au fauchoir une grande partie des Rophites. I) plus, cet insecte vole beaucoup plus rapidement que l'Abeille, sans s'élever au-dessus des fleurs durant son vol. Toutes ces raisons allant dans le même sens m'auraient amené à sous-estimer dans de très grandes proportions l'importance de la population de Rophites si mon étude avait été effectuée à l'aide du seul fauchoir.

Pour le moment, il ne semble pas y avoir d'autres méthodes valables que celle de l'observation pure et simple. I,es pièges aspirants qui, dans d'autres recherches écologiques, donnèrent d'excellents résultats, sont ici inopérants. En effet, les Abeilles sont de trop grande taille pour pouvoir être capturées en nombres significatifs et il ne semble pas possible d'augmenter la puissance des aspirateurs, étant donné qu'il doit en exister sur le terrain un nombre relativement élevé pour que les résultats puissent être pris en considération.

I.a méthode d'observation à vue elle-même devra, dans les années à venir, faire l'objet d'une étude sérieuse. Combien faut-il faire de recensements par jour et de quelle importance pour avoir une estimation juste à tant pour cent de la population réelle d'un champ donné, voilà la question que nous devons nous poser. A cette question, une analyse statistique des résultats pourra nous donner une réponse, mais il était indispensable d'effectuer au préalable les remarques que nous venons de faire afin d'éliminer les causes d'erreur apparentes.

I)ans quelques années, nous en serons peut-être à donner, comme le font des phytoecologistes une estimation exacte en heure de travail de plusieurs méthodes susceptibles de donner une information exacte avec une erreur standard de I 5 p. IOO sur la composition de la population végétale d'un bois de io hectares (LINDSEY et coll. I958).

Un autre procédé doit être signalé qui permet théoriquement de connaitre l'importance de la population d'une espèce donnée, non plus dans le cadre d'un champ, mais dans un biotope donné.

En effet, si on capture un certain nombre d'animaux dans un biotope considéré comme clos et que ces animaux soient relâchés après avoir été marqués de manière indélébile, une formule basée sur la proportion d'animaux marqués repris lors d'une seconde capture permet d'estimer la population totale du biotosope considéré.

Soit la formule $\mathrm{P}=\mathrm{NH} / \mathrm{P}$ où $\mathrm{P}$ est la population inconnue, N le nombre d'animaux marqués, $H$ le nombre d'animaux capturés lors de la seconde capture et $\mathrm{R}$ le nombre d'animaux marqués trouvés lors de cette seconde capture.

Cette méthode est fort ancienne, puisqu'elle fut appliquée dès I 896 par PETERSEN pour étudier les populations de Carrelets de la Baltique au large du Danemark. Depuis, elle a été employée à de-très nombreuses reprises, mais surtout pour l'étude de populations de vertébrés. Elle est pourtant parfaitenent applicable aux insectes. J'ai eu l'occasion d'en opérer une vérification dans les circonstances suivantes :

Au cours d'un marquage au moyen de radiosotope, sur lequel j'aurai l'occasion de revenir, j'ai marqué la totalité des butineuses d'une ruche; cette ruche se trouvant dans une vallée assez étroite, un très petit nombre d'Abeille pouvait provenir de ruches extérieures à cette vallée ou ses environs immédiats. Dans ce milieu 
quasi “fermé " se trouvaient 30 ruches, or, lors de la reprise, sur 2.442 butineuses, il s'en trouvait $8 \mathrm{I}$ de marquées. Soit un rapport de 30,9 qui nous indique un nombre de ruches très voisin de celui que nous connaissions.

Dans le cas des Abeilles, il est plus facile évidemment de dénombrer directement les colonies, mais, en ce qui concerne les Apides sauvages, on conçoit tout ce que peut apporter la méthode.

Faisons tout de suite d'ailleurs quelques remarques; un certain nombre de conditions sont requises pour l'emploi de cette méthode de dénombrement. RrCKER (r948) les a ainsi formulées en ayant en vue l'étude des populations de poisson.

La mortalité doit être la même parmi les animaux marqués et non marqués. C'est le cas des Abeilles marquées avec un radioisotope, une étude préalable (CourTors et LEConTe I959) nous l'ayant démontré. Les individus marqués ne doivent pas perdre leur marque. Nous sommes dans ce cas. Les individus marqués doivent être capturés aussi facilement que les autres. Ia question ne se pose généralement pas dans le cas des insectes, il n'en est pas de même avec certains vertébrés. Le premier marquage est effectué à la suite d'un piégeage et lors de la reprise, l'expérience du piège fausse les résultats. Les Animaux marqués doivent être mélangés, au hasard, avec les autres. Dans le cas de l'Abeille, ce que nous connaissons du comportement de butinage nous incite à la plus grande prudence. Cependant, dans la plupart des cas, 80 p. Ioo de la population de butineuse ne s'écarte pas à plus de $\mathrm{I} \mathrm{km}$ 5oo de la ruche. I)ans ce périmètre il est possible de prospecter également toutes les sources de nourriture et d'y faire des prélèvements d'égale importance.

Enfin, il ne doit pas y avoir trop de fluctuations dans l'importance de la population durant le recensement. Un taux de naissance trop élevé ou des migrations peut entraîner des erreurs importantes.

En ce qui concerne l'Abeille, nous pouvons remarquer que le nombre d'individus marqués parmi les butineuses reste constant pendant plusieurs jours, les mortalités étant compensées par les sorties de nouvelles butineuses également marquées du fait du principe du marquage. I 'ailleurs, il est possible d'éliminer cette cause d'erreur en employant une formule légèrement différente. Il s'agit de la formule de SchNaBEI. (1938). Ici $\mathrm{P}==(\mathrm{AB}) \mathrm{C}$. P reste l'inconnue à déterminer. C'est le nombre total de recaptures et $(A B)$ est la somme des produits journaliers des captures et des individus marqués présents dans ces captures. Dans ce cas, pour que la méthode ait intérêt un supérieur à celle de PeTfrsex, il importe que les individus marqués ou non, capturés soient relâchés.

Parmi les utilisations entomologi(ques de ces procédés, citons les travaux sur Isétsé de Jackson (I937-I939). Michiarkr (I953) a étudié également de la sorte une population de Megachile.

\section{TECHNIQUES DE MARQUAGE}

Un grand nombre de problèmes posés par les Abeilles butineuses demandent, pour leur solution, la possibilité d'un marquage permettant, soit de reconnaître tel ou tel individu, soit de reconnaître l'ensemble des individus en provenance d'une ruche donnée. 
L'histoire du marquage des butineuses est certainement aussi vieille que celle de l'exploitation de l'Abeille par l'homme. L'on trouve la description d'un tel procédé dans le traité d'agriculture de CoLUMEI,LA, écrivain latin du premier siècle et plusieurs exemples analogues peuvent être trouvés dans les traités d'ethnographie.

L'utilisation à des fins scientifiques d'un procédé de marquage est certainement loin d'être aussi ancienne, cependant, durant les trente dernières années, un certain nombre de chercheurs ont été amenés à imaginer des procédés de types divers.

Nous passerons en revue, tout d'abord, quelques procédés permettant de marquer individuellement des abeilles et de les reconnaitre facilement. GritTay (Igo6) a mis au point une méthode qui permet une reconnaissance facile, mais qui parait d'une application délicate et lente. Après avoir capturé 1'Abeille, ce chercheur écrivait tout simplement un chiffre au moyen d'un pinceau très fin et d'encre blanche, sur le thorax. Vox Frisch a considérablement perfectionné ce procédé en remplaçant les chiffres arabes employés par GIrTAY par un code bien plus simple à appliquer et facile à lire. On trouvera une description complète de ce marquage dans différentes publications, par exemple : VON FRISCH (I922-I923).

La peinture est composée de pigments employés par les peintres pour la préparation des peintures à l'huile. Ces pigments sont mélangés à une solution de gomme laque (Weisser Schellack) dans de l'alcool.

Cinc couleurs sont employés par Vor Frisch selon le code suivant:

Sur la partie antérieure du thorax : Blanc $=I$, Rouge $=2$, Orangé $=3$, Jaune $=4$, Vert $=5$.

Sur la partie postérieure du thorax : Blanc $=6$, Rouge $=7$, Orangé $=8$, Jaune $=9$, Vert $=0$.

Sur l'abdomen : Blanc $=$ Ioo, Rouge $=200$, Orangé $=300$, Janne $=400$, Vert $=5$ oo.,

On peut ainsi numéroter 5099 Abeilles et les distinguer les unes des autres. La lecture se fait en commençant par le côté gatuche de l'animal. Ainsi 29I se lira sur une Abeille présentant un point rouge sur l'abdomen, un point jaune sur la partie postérieure du thorax et à gauche, un point blanc sur la partie antérieure et à droite. Un certain nombre de variantes ont été introduites par la"suite par différents auteurs ; c'est ainsi que PARK (Ig29) n'a pas utilisé le même systlième de notation à cause de la difficulté que présente, pour la lecture, l'emploi de deux couleurs différentes sur le thorax.

PARK s'est contenté d'utiliser la combinaison de sept couleurs différentes et du marquage sur le thorax et sur l'abdomen. Cet auteur a également mis au point un dispositif destiné à faciliter le repérage des butineuses à l'entrée de la ruche. Ce dispositif consiste en un tunnel divisé en passages étroits, recouverts de plaque de verre mobile. Ce dispositif permet d'obtenir les butineuses et aussi de saisir celles que l'on désire étudier, mais il est la cause d'embouteillages et de perturbations assez ennuyeuses. PARK a également réalisé d'autres dispositifs constitués par des brosses ayant le poil dirigé vers le bas ; ces dispositifs ont l'intérêt d'obliger les butineuses à montrer leur dos.

RibBANDs (I960), contrairement aux auteurs précédents, ne marque pas l'Abeille au travail ou pendant qu'elle se nourrit, mais opère à l'aide d'une légère anesthésie provoquée par du chloroforme. Il a, bien entendu, vérifié que cette anesthésie n'altérait en rien le comportement de butinage. Le marquage thoracique a été éliminé par RIB- 
BANDS, comme présentant l'inconvénient de détruire les poils de cette partie du corps et réduire ainsi la capacité de récolte de pollen.

Le tergite du deuxième segment abdominal visible a finalement été sélectionné pour opérer les marquages, comme étant la plus grande surface ininterrompue de tégument, ce qui permet d'éviter d'enduire de peinture les membranes intersegmentales ou des poils collecteurs de pollen.

Les points de peinture de très petite taille sont à éviter, car ils sont facilement perds. Finalement, un système basé sur les lettres de l'alphabet schématisées fut élaboré. Deux critères servirent à créer ces symboles : la facilité d'exécution et l'impossibilité de confusion entre deux figures, même en cas d'altération. L'auteur a fait usage de peinture cellulosique, en utilisant uniquement des teintes pâles : blanc, jaune, rose ou bleu. Après avoir reçu leur marque distinctive, les Abeilles sont placées dans une assiette de porcelaine, au soleil, et le très faible pourcentage qui parait incapable de prendre son vol est éliminé.

J'arrêterai ici l'énumération des procédés permettant un marquage individuel, car les autres auteurs dont je connais les travaux n'ont guère modifié ces méthodes, ou ne l'ont pas signalé de manière très explicite dans leurs publications. Personnellement, j'ai utilisé à plusieurs reprises des procédés très analogues avec satisfaction. On peut seulement dire, au sujet des différents codes, qu'il s'agit d'une question très personnelle et qu'un code doit être adapté aux recherches effectuées, et aussi à l'utilisateur. En ce qui concerne les peintures employées, il semble bien que les solutions dans l'alcool de gomme laque et de pigment peuvent être avantageusement remplacées par les multiples peintures cellulosiques que l'on trouve actuellement, en particulier les peintures utilisées par les constructeurs de modèles réduits d'avions ou de bateaux donnent toute satisfaction, à condition d'être ajustées à la consistance souhaitable par évaporation ou par adjonction de diluants. Enfin, les pinceaux utilisés par la plupart des auteurs peuvent être remplacés par de très fines tiges de verre, plus faciles à nettoyer et moins coûteuses.

Toutes les peintures ayant cependant tendance à disparaître, un procédé plus durable a été mis au point (MICHENER et coll. I955). Les ailes sont teintes avec une solution saturée alcoolique ou acétonique de méthyl ou de méthyléosine. Cette teinture n'étant pas soluble dans l'eau persiste très longtemps; en cas de doute, il est possible, en plaçant un papier filtre humecté d'alcool sur l'aile, d'acquérir une certitude. Malheuretusement, ce procédé ne permet de reconnaître qu'un très petit nombre d'individus.

Nous étudierons maintenant les procédés utilisés pour le marquage en masse d'une grande quantité d'ouvrières, soit l'ensemble de la population d'une ruche, soit l'ensemble des butineuses présentes sur une surface quelconque.

Il est évidemment impossible de marquer au moyen de peintures appliquées individuellement une population de plusieurs dizaines de milliers de butineuses; aussi, a-t-on cherché un procédé permettant un marquage massif. SingH (I950) a utilisé une peinture, en aérosol, sans aucun succès. Parmi les méthodes les plus efficaces, signalons d'abord la technique de NAIRN (I953) ; cet auteur a utilisé un mélange de talc et de poudre d'aluminium, contenu dans un dispositif spécial, à l'entrée de la ruche; ce mélange pouvant d'ailleurs être coloré de différentes manières. L'Abeille en prenant son vol, se saupoudre de ce mélange et peut être reconnue dans les champs. 
Les essais que j'ai effectués avec cette méthode ne m'ont pas donné entière satisfaction. Outre que la présence du dispositif entraîne quelques perturbations dans le vol des butineuses, le marquage n'est pas suffisamment net pour la plupart des butineuses, pour d'autres, au contraire, la dose est excessive et paraît constituer une gêne. Ce prođécé, très simple d'application cependant, ne convient que si on désire une indication approximative sur la répartition des butineuses des ruches étudiées.

Musgrave (I949-I950) a mis au point un procédé de marquage à l'aide de pigments fluorescents. Ici, on ne procède plus au marquage des butineuses d'une ruche donnée, mais au marquage de celles qui visitent une zone définie. Pour cela, les fleurs sont saupoudrées avec du talc et de la fluorescéine, deux parties pour une. Les butineuses qui visitent ces fleurs retiennent grâce à la pilosité de leur corps, suffisamment de fluorescéine pour qu'une fluorescence puisse être observée à 1'œil nu. Malheureusement, cette méthode, pour être pratiquable sur le terrain, implique le sacrifice des butineuses qu'il faut immerger pendant quelques minutes dans une solution à 0 , I 5 p. Ioo de potasse.

Il devient donc très difficile, surtout s'il existe un nombre élevé de colonies à proximité des fleurs marquées, de retrouver un nombre significatif de butineuses. Un essai m'en a convaincu.

Au contraire, l'utilisation de pigments fluorescents, telle qu'elle a été pratiquée par différents auteurs (Smith I95I, Johanson I959) paraît plus intéressante. Ces auteurs saupoudrent avec abondance une ou plusieurs fleurs et étudient la dispersion du pigment dans les fleurs avoisinantes. JoHANson prélevait les fleurs et les examinait au laboratoire sous lumière ultraviolette. La dispersion du pigment pouvant donner une indication sur la dispersion potentielle du pollen du fait des insectes pollinisateurs. SMrTh opérait de même, à cela près qu'il ne prélevait pas les fleurs, mais les inspectait " in situ ", de nuit, à l'aide d'un projecteur UV portatif.

Ces méthodes permettent de détecter de très faibles transports de substances et semblent supérieures à celles utilisées par STEPHENS et FINKNER (I953) et THIES (I953) qui marquaient les fleurs à l'aide de bleu de méthylène. Le marquage en vue d'observer le transport du pollen peut également être effectué par voie interne. C'est ce qui a été réalisé sur Luzerne par DEMarr Y (1955). Du Phosphore 32 a été introduit dans la plante par les racines ; il s'est rapidement distribué dans la plante, de telle sorte qu'au bout de 9 jours il devint possible de détecter de manière satisfaisante la présence de Io grains de pollen en provenance de la plante marquée. Il fut ainsi possible de mettre en évidence que le rayon de dispersion était maximum de Io mètres.

Revenons aux pigments fluorescents, pour signaler qu'il est aussi possible de marquer les Abeilles à la sortie de la ruche avec un dispositif analogue à celui utilisé par Narrs et déjà signalé. Ce pigment peut être détecté sur les fleurs, la nuit, grâce au projecteur portable à UV ; il peut être détecté également sous lumière noire sur les Abeilles. Différents auteurs ont employé cette technique (JohAssson I959) (Smith et Townsend I958) (Smr'th r958).

La question du marquage massif d'une population de butineuses n'a cependant été résolue d'une manière satisfaisante qu'avec l'utilisation des radio-isotopes. Ce type de marquage, déjà utilisé pour étudier le déplacement des animaux les plus divers est d'ailleurs facilité, dans le cas de l'Abeille, par de continuels échanges de nourriture entre les individus. NIXON et RIBBANDS (I952) ont étudié ces échanges, en faisant in- 
gérer à quelques ouvrières du sirop de sucre contenant du phosphore 32 , ils ont ainsi mis en évidence la rapidité de la transmission entre les membres de la colonies.

En collaboration avec la Section d'Application de Radioéléments du Centre d'Études nucléaires de Saclay, j'ai mis au point une méthode de marquage basé sur cette transmission (CourtoIs et LeCOMTE I958-I959 a) (LECOMTE I960). Notre choix, pour diverses raisons exposées dans les publications signalées, s'est porté sur l'Or r 98 dont la période de 2,68 jours permet d'éviter les contaminations et annule pratiquement les risques d'accidents.

Une dose d'un microcurie par abeille pernet une reconnaissance facile sur le terrain pendant trois jours. Cette opération peut alors être renouvelée, ce qui porte la durée de travail à 6 jours, période qui nous parait largement suffisante. La distribution du radioisotope s'effectue grâce à une suspension d'or colloïdal dans un sirop de sucre placé dans un nourrisseur à l'intérieur de la ruche.

La reconnaissance des Abeilles marquées est parfois possible en approchant le détecteur de la butineuse ; mais seulement si cette dernière est suffisamment immobile. Le plus souvent, il est plus avantageux de capturer l'ouvrière avec un filet à insecte et de la relacher après l'avoir examinée. Cette dernière méthode permet, en particulier, d'étudier les butineuses de la cîme des arbres et permet d'utiliser une constante de temps élevée de 1'appareil détecteur (détecteur portatif à scintillation) ce qui accroît sa sensibilité. D'autre part, il a été vérifié que la dose de radioactivité reçue au cours de l'opération était très inférieure à celle susceptible de provoquer des troubles du comportement de l'ouvrière (Courtors et LECOMTE I959 b). La technique du marquage par radio-isotope a été depuis employée par un certain nombre d'auteurs. Ceux-ci ont employé un autre radio-isotope, le Phosphore 32 qui possède une période de I4 jours. 1'Or leur semblant décroître trop rapidement. Grâce à ce procédé, un certain nombre de travaux intéressants (LEVIN I960-I96I, $a, b$,) (LEE I96I) ont pu être réalisés. Je pense cependant que 1'or 198 offre une sécurité d'emploi bien plus grande et que, dans les régions à peuplement relativement dense où nous travaillons, ce dernier facteur doit être pris en considération.

Une autre méthode permettant un "marquage " de masse est celle qui consiste à profiter d'une mutation suffisamment rare pour ne pas exister dans les diverses colonies incontrôlées du secteur étudié et facilement identifiable. La mutation "Cordovan " a été ainsi utilisée dès I954 par 'TABER pour étudier 1'accouplement de la reine d'Abeille. Elle a été utilisée pour étudier le comportement de butinage par LEVIX (I959-I96I $a, c)$ et par TABER (I960) ; LEVIX (I960) a effectué une comparaison des deux méthodes, emploi d'une mutation, marquage par radio-isotope et semble avoir obtenu des résultats comparables. La méthode utilisant les mutations ne nécessite pas d'appareillage coûteux ou des connaissances spéciales, par contre, on court le risque d'opérer dans une région où se trouve déjà une ruche contenant à l'état pur ou hybride des abeilles mutantes. Le fait d'utiliser ces abeilles dans un but expérimental contribuera d'ailleurs à répandre le gène dans les exploitations commerciales et diminuera l'intérêt de la méthode. Signalons aussi que le comportement des mutants semble identique à celui des ouvrières normales.

Enfin, le gène peut exister chez les différentes races d'abeilles connues. I1 est donc possible d'obtenir différentes souches de Cordovan différents par le nombre de bandes abdominales et d'opérer une distinction entre elles. Bien qu'il soit 
théoriquement possible d'opérer des marquages avec des radio-isotopes différents et de les distinguer les uns des autres, aucune tentative de cet ordre n'a été effectuée à ce jour, à ma connaissance. Les deux méthodes : mutation et radio-isotope se complètent donc fort heureusement et doivent, judicieusement employées, faire progresser nos informations sur le comportement de butinage.

\section{TECHNIQUES DIVERSFS}

Il peut être intéressant de distinguer parmi les butineuses, celles qui rapportent de l'eau à la ruche de celles qui rapportent du nectar. PARK (I929), par pression, fait dégorger sa récolte à l'Abeille sur une feuille de papier filtre ; le nectar laisse une trace ressemblant à une tache d'huile, alors que l'eau ne laisse rien. Les butineuses qui se seraient chargées de miel au départ de la ruche dégorgent un liquide qui laisse une tache transparente, mais qui durcit le papier (PARKER I926).

I,e volume du jabot, et donc de la récolte, peut être intéressant à connaître, car la fréquence d'Abeilles, à jabot bien rempli, renseigne sur l'abondance de la sécrétion nectarifère. HAMMER (I953) estime le volume du jabot par comparaison avec de petites boulettes de cire dont le volume est connu.

La richesse en sucre du nectar est très souvent apprécié en plaçant le contenu du jabot dans un réfractomètre. Cette méthode semble universellement adoptée, comme beaucoup plus simple que celle qui consiste à prélever le nectar directement sur les fleurs depuis que PARK (r933) a réfuté la théorie selon laquelle les butineuses concentraient le nectar durant le vol de retour. Parmi les auteurs ayant employé cette méthode, citons : VAxseli, (I942) et HAMmer (I953). Cependant, la concentration en sucre du contenu du jabot ne correspond pas exactement à celle du nectar. Une série d'essais effectués en cagette nous a renseigné sur les variations observables.

Les Abeilles, après un jeune d'une heure, sont nourries avec un sirop de sucre de concentration connue, et sacrifiées $I / 2$ h après.

On s'aperçoit alors que les concentrations de sucre en pour cent du contenu du jabot s'écartent parfois notablement en plus ou en moins des concentrations initiales du sirop de sucre. La moyenne obtenue avec I 5 butineuses donne un résultat différent cle \pm 5 p. Ioo au maximum, ceci aussi bien pour les concentrations faibles que pour les fortes.

Il y a donc lieu de ne pas prendre trop à la lettre les résultats obtenus par cette méthode qui constitue essentiellement un moven rapide d'obtenir un ordre de grandeur à ro p. Ioo près.

Une autre récolte peut être étudiée qui fournit des renseignements précieux sur les plantes visitées, il s'agit du Pollen.

Selon les espèces, le pollen est récolté dans la Corbeille située sur les parties postérieures ou sur une Brosse de poils raides situés sur la face ventrale de l'abdomen. Dans l'un et l'autre cas, les pollens peuvent être collectés sur l'individu, à l'entrée de la colonie, ou dans les cellules. Ils sont alors identifiés et donnent de précieux renseignements. En ce qui concerne 1'Abeille domestique, on trouvera une bibliographie détaillée dans la thèse de I,OUvEAux (I958). 
Les grains de pollen existent aussi dans le nectar et peuvent renseigner sur la provenance de celui-ci. En ce qui concerne l'analyse des grains de pollen contenus dans le jabot, on peut consulter MaUrizio (I949).

L'analyse pollinique des miels est une technique largement répandue et il existe à ce sujet une très abondante littérature. Contentons-nous de signaler une revue récente (MAURIzIO I960).

\section{CONCLUSION}

Les différentes techniques utilisables pour l'étude des popt1lations d'Apides en ayant surtout en vue leur activité pollinisatrice ne se limitent pas à celles que nous venons d'exposer. On peut, par exemple, trouver d'utiles renseignements dans différents travaux (LINSLEY et coll. I952) (MICHENER et coll. I955). D'autre part, 1'intérêt économique de ces recherches, joint à celui que leur portent nombre de chercheurs dégagés de ces préoccupations, font que la question est en continuelle évolution.

L'ère de la simple observation est révolue et la complexité des problèmes écologiques ou éthologiques rend nécessaire l'utilisation de méthodes de plus en pltss rigoureuses et efficaces.

Reçu pour publication en mai 1962

\section{SUMMARY}

TECHNIQUES FOR STUDYING POPULATIONS OF INSECT POLLINATORS

The study of populations of insect pollinators, chiefly the Apidae, calls for the employment of certain techniques. Those examined in this study are concerned essentially with the measurement of population densities and with the marking of individuals and collections of individuals occurring in these communities.

\section{RÉFÉRENCES BIBLIOGRAPHIQUES}

Courtois G., Lecomte J., I958. Sur un procédé de marquage des Abeilles butineuses au moyen d'un radioisotope. C.R. Acad. Sc., 247, I47-I49.

Courtois G., Leconte J., i959 a. Marquage d'Abeilles au moyen d'or radioactif. Int. J. of App. Rad. and Isolopes., 5, 265-268.

Courtors G., Lecomte J., I959 b. Sur la résistance au rayonnement gamma de l'Abeille ouvrière. Ann. abeilles, 2, 285-290.

Demarly Y., 1955. Geneva Conférence Paper p. 382.

FrISCH I. von., I922. Methoden sinnesphysiologischer und psychologischer Untersuchungen an Bienen. Abderhalien's Handb. biol. Arbeitsmethoden, Berlin und Wien., I22-78.

FRISCH K. von, 1923. Über die "Sprache " der Bienen. Iena, I86 p.

Giltay., Igo6. Uंber die Bedeutung der Krone bei den Blüten und über das Farbenunterscheideungvermögen der Insekten II. Jarhrb. wissensch. Botanik.

Hambleton J., 1925. The effect of weather upon the change in weight of a colony of bees during the honey flow. Bull. U.S. Dep. Agric. n ${ }^{\circ} \mathrm{I} 3395^{2} \mathrm{p}$.

Hammer O., 1953. Observations sur la sécrétion nectarifère chez le Romarin (Romarinus officinalis). Revue Fir. Apiculture, février, 397-403.

JAckson C. H. M., 1937. Some new method in the study of Glossina morsitans. Proc. Zool. Soc. Lond., 8I I -96 .

Jackson C. H. M., I939. The analysis of an animal population. J. Animal Ecol., 8, 238-46.

JAmieson C. A., I950. Investigations at the Bee Division. Can. Bee Jour., 58, 2 20-22. 
Jortaxssov I. S. K., I959. Tracking honey bees in cotton fields with fluorescent pigments.J. Econ. Ent 52, 4, 572-577.

LeConte J., rg60. Observations sur le comportement des Abeilles butineuses. Ann. Abeille., 3, 317-27.

LEE W. R., I96I. The nonrandom distribution of foraging honey bees between apiaries. J. Econ. Ent., 54, 5, 829-930.

LEvIN M. D., I959. Distribution patterns of young and experienced honey bees foraging on Alfafa. J. Econ. Ent., 52, 969-7r.

LEvis M. D., I960. A comparaison of two methods of mass marking foraging Honey Bees. J. Econ. Ent., 53, 695-9o.

LEvin M. D., r96 $a$. Distribution of foragers from IIoney Bee colonies placed in the middle of a latrge field of Alfafa. J. Econ. Ent., 54, 431-36.

LEVIN M. D., I961 $b$. Interactions among foraging Iloney Bees from diffreent apiaries in the same field. Insectes Sociaux, 8, 195-201.

Levis M. D., ig6i c. The dispersion of ficld Bees on Alfafa in relation to a neighboring apiary. J. Econ. Ent., 54, 482-84.

LiNDSEY A. A. et coll,, I958. Field efficiencies of forest sampling methods. Ecology', 39, 3, 428-444.

LiNSLEY F. G., I946. Insect pollinators of Allafa in California. J. Econ. Ent., 39, I i8-29.

Louveaux J., 1958. Recherches sur la récolte du pollen par les abeilles (.1pis mellifica I..). Ann. Abeille, 1, 206.

Maurizio A., 1953. Weitere Untersuchungen an Pollenhïschen Beih. Schweiz. Bienen-Zg., 2, I3, 4S6.

Maurizio A., 1960. Bienenbotanik (in Jiene und Bienenzucht.) Munich, p. 68-1o4.

MiChenenR C. D., I953. The biology of a leafcutter Bee (Megachile) brvis and its associates. U. of Kansas Science Bull., 35, no ${ }^{\circ} 6$, I659-I 748.

Miciener C. 1), et coll., I955. Additional techniques for studying the behavior of wild bees. Insectes Sociaikx., 2, 237-246.

MUsgrave A. J., I949. The use of a duorescent material for marking and detecting inserts. Canad. Enl., $81,7,173$.

Muscrave A. J., I950. A note on the dusting of crops with fluorescein to mark visitingr bees. Canalian Lint., 82, 9, 195-6.

NAiry R. G, i953. A new method of marking bees. New Z. J. Agric, 86, 6.

Nixon H. L., Ribbaxps C. R., 1952. Food transmission in the honeybee community. Proc. Roy. Soc. N., 140, $43-50$.

PARK O. W., I928. Time factors in relation to the acquisition of food by the honeybee. Res. Bull. I a. agric. Exp. Sta., no 108, I83-225.

PARK O. W., i933. Studies on the rate at which honeybee ripen honey. Eicon. Ent., 26, r88-93.

PARker R. L., I926. The collection and utilisation of pollen by the honeybee. 11em Comell. agric. E. Sta., $\mathrm{n}^{0} \mathbf{9 8}, \mathrm{I}-55$.

J'ercival M., 1950. Pollen presentation and pollen collection. New Phytol., 49, I, 40-63.

P'ercival M., 1959. The présentation of pollen in certain angiosperms and its collection by Apis mellifica. New Phytol., 54, 3, 353-368.

I'ETERSEN C. G., I8g6. The yearly immirration of young into the Limfjord from the german sea. Rep. Danish Biol. Si., 6, г-48.

Ribbands C. R., I949. The foraging method of individual honeybees. J. Anim. Ecol, 18, 47-66.

Ricker W. E., 1948. Method of estimating vital statistics of fisch populations. Indiana T. Pubbl. Sci. Ser. no 15 .

SCIXABEL Z. E., 1938. The estimation of the total fisch population of a liake. American Malh. Monlh., 65, $3+S^{-}-52$.

ScirUt L., I952. Untersuchungen über den Linfluss meteorologische Elemente auf das Verhalten der IIonirbienen. Z. Verg. Physiol., 34, 258-77.

SiNgrr S., I950. Behaviour studies of honcybees in gathering nectar and pollen. Bull. Cornell Expp. st., $10^{\circ} 288, \mathbf{I}-59$.

SurTir M. V., I958. The use of tuorescent markers as an aid in studying the foraging behaviour of Honeybees. Proc. Ioth Internat. Congr. Entonnol, 4, 1063.

Suith M. V., Towsend G. F', 1951, A technique for mass-marking honeybees. Canadian Ent., 83, 346-48.

Suith M. V., Towsend G. F., 1952. A method of measuring pollinator populations on fields crops. Canadian Ent., 74, 314-16.

Stephens S. G., Finkner M. D., 1953. Natural crossing in cotton. Licon. Bot., 7, 257-69.

TABER S., 1954. The frequency of multiple mating of queen Iloneybees. J. Econ. Ent., 47, $995-8$.

TABER S., IgGo. Estimation of total honey bee populations using a known population of marked bees. J. Econ. Ent., 53, 993-95.

TuIEs S. A., r953. Agents concerned with natural crossing of cotton in Oklahoma. Agron. J., 45, I0, 48I-84.

VANSELl G. H., I942. Factors affecting the usefulness of honeybees in pollination. U.S. D. A. Circ., n ${ }^{\circ} \mathbf{6 5 0}$, $3 \mathrm{I}$ p.

Vaxsell G. H., Tond F. E., I 946 . Alfafa tripping, J. Amer. Soc. Agr., 38, 470-488. 